\title{
Study on Detecting the Gelling of Preserved Eggs by Using Dielectric Method
}

\author{
Cheng-Han Li ${ }^{1}$, Cheng-Chu Hung ${ }^{1}$, Ching-Wei Cheng ${ }^{2}$ \\ ${ }^{1}$ Department of Bio-industrial Mechatronics Engineering/National Chung Hsing University \\ No.145 Xingda Rd., South Dist., Taichung City 402, Taiwan (R.O.C.) \\ L634259@gmail.com; ackt223@gmail.com \\ ${ }^{2}$ College of Intelligence/National Taichung University of Science and Technology \\ No.129, Sec.3, Sanmin Rd., North Dist., Taichung City 404, Taiwan (R.O.C.) \\ cwcheng@nutc.edu.tw
}

\section{Extended Abstract}

Preserved eggs are mainly marinated from fresh duck eggs. The marinated duck eggs are much easier to keep than fresh duck eggs and have abundant protein and amino acids [1]. Preserved eggs have a unique smell and taste that can promote appetite and are well-known traditional Chinese foods in Asia.

According to statistics from the Taiwan government and the Duck Association Republic of China, Taiwan's annual output of fresh duck eggs was about 440 million pieces, and the annual total export value was about 56 million US dollars. The preserved eggs utilize the pickling of alkaline substances to cause protein chemical reactions inside the duck eggs to solidify. In general, the production method of preserved eggs can divide into three techniques: coating method, immersion method, and combined methods [2-4]. After the interaction between the duck egg and the alkaline substance, the duck egg will become a preserved egg. The standard preserved eggshell is easy to remove from the preserved egg surface, and the appearance of the preserved egg albumen is smooth and like the jelly of tan gel. Pine pattern road is also known as needlelike crystal, which is formed by the egg yolk component moving to egg albumen. The egg yolk will turn dark green or grass green solidified body in different shades. For preserved eggs with incomplete gel, a thick brown liquid may flow out after the eggshell remove, or the eggshell is easy to stick to the egg albumen surface and not easy to remove. It also emits a pungent smell, which may cause nausea.

The detection of preserved eggs is mostly checked by workers. Traditionally, the degree of vibration of the preserved eggs is tapping the preserved eggs with a finger and feel the amplitude of the oscillation to determine the degree of gel of the preserved eggs. This process requires a long term experience of training. This study uses the dielectric technique to measure the preserved eggs such that a scientific and objective classification method for the quality of gelatin inside the egg provides. The non-destructive dielectric technique detected the preserved eggs with two types of different angles plate electrodes, and the dielectric capacity of the preserved eggs is measured at a low-frequency signal of $100 \mathrm{~Hz}$ to determine the degree of internal gelation. The results show that the confusion matrix, area under the curve of ROC (AUC ROC), and the Kappa value show that at $100 \mathrm{~Hz}$, the accuracy of the parallel plate electrode for preserved egg gelling identification is $85.7 \%$, the Kappa value is 0.714 , and the AUC ROC is 0.902 . The classification accuracy of the plate electrode with an angle of 60 degrees is $76.1 \%$, the Kappa value is 0.53 , and the AUC ROC is 0.76 . The results show that the parallel plate electrode can more accurately judge the gel state of the whole egg than a plate electrode with an angle of 60 degrees.

\section{References}

[1] H. M. Chang, C. F. Tsai and C.F. Li, "Changes of Amino Acid Composition and Lysinoalanine Formation in AlkaliPickled Duck Eggs," J Agric Food Chem., vol. 47, no. 4, pp. 1495-1500, 1999.

[2] C. Ye, "Technique of Preserved Egg Processing and Edible Security," Agricultural Science \& Technology and Equipment, vol. 8, pp. 62-63, 2015.

[3] X. Zhang, A. Jiang, M. Chen, H. W. Ockerman and J. Chen, "Effect of different alkali treatments on the chemical composition, physical properties, and microstructure of pidan white," J Food Sci Technol., vol. 52, no. 4, pp. 22642271, 2015. 
[4] Y. Zhao, Z. Chen, J. Li, M. Xu, Y. Shao and Y. Tu, "Changes of microstructure characteristics and intermolecular interactions of preserved egg white gel during pickling," Food Chem., vol. 203, no. 15, pp. 323-330, 2016. 